\title{
Rola-bostas (Coleoptera: Scarabaeidae: Scarabaeinae) de florestas e pastagens no sudoeste da Amazônia brasileira: Levantamento de espécies e guildas alimentares
}

\author{
Ricardo J. SILVA ${ }^{* 1,2}$, Fabricio COLETTI ${ }^{3}$, Diogo A. COSTA ${ }^{2,4}$, Fernando Z. VAZ-DE-MELLO ${ }^{5,6}$ \\ 1 Universidade Federal da Grande Dourados, Pós Graduação em Entomologia e Conservação da Biodiversidade. Rodovia Dourados - Itahum, km 12, Cx. Postal: 364, 79.804-970, Dourados - MS, Brasil. \\ 2 Universidade do Estado de Mato Grosso, Centro de Pesquisa, estudos e desenvolvimento agro-ambiental. Rodovia MT 358, km 07, Jardim Aeroporto, Tangará da Serra MT. CEP 78300-000, Brasil. \\ 3 Universidade do Estado de Mato Grosso, Pós Graduação em Ambientes e Sistemas de Produção. Rodovia MT 358, km 07, Jardim Aeroporto, Tangará da Serra - MT. CEP 78300-000, Brasil. \\ ${ }^{4}$ Universidade Federal de Viçosa, Pós Graduação em Entomologia, Departamento de Entomologia. Viçosa - MG. CEP 36570-000, Brasil. \\ ${ }^{5}$ Universidade Federal de Mato Grosso, Departamento de Biologia e Zoologia, Instituto de Biociências, Av. Fernando Correa da Costa, 2367, Boa Esperança, Cuiabá - MT. CEP \\ 78060-900, Brasil. \\ ${ }^{6}$ Julho de 2013 a junho de 2014: Muséum National d'Histoire Naturelle, UMR 7205, Département Systématique et Évolution, Entomologie, case postale 50, 57 rue Cuvier, \\ F-75231 Paris cedex 05, France. \\ *Autor correspondente: ricardojosesilva11@gmail.com.
}

\section{RESUMO}

Os besouros rola-bostas são insetos copro-necrófagos e utilizam primariamente fezes e carcaça de mamíferos como fonte de alimento e nidificação. Estes insetos são sensíveis à modificação do habitat principalmente relacionados à cobertura da vegetação. A substituição da floresta Amazônica por pastagem altera drasticamente o habitat das espécies nativas e acarreta redução da riqueza de espécies e modificação na composição das assembleias locais. O objetivo deste trabalho foi compreender os efeitos da substituição da floresta nativa por pastagem introduzida na riqueza, abundância, composição de espécies e estrutura das guildas alimentares dos besouros rola-bostas no sudoeste da Amazônia brasileira. Foram coletados 10.073 indivíduos de besouros rola-bostas pertencentes a 84 espécies e 22 gêneros. As florestas (seis fragmentos de floresta secundária) apresentaram 71 espécies em sua maioria com abundância intermediária. As pastagens (áreas vizinhas onde a floresta original foi substituída por pastagens introduzidas) apresentaram redução significativa no número de espécies, e composição de espécies diferente. Nas florestas a maioria das espécies foi considerada generalista, enquanto as pastagens apresentaram maior abundância de espécies coprófagas o que demonstra uma modificação na guilda alimentar causado pela substituição da floresta por pastagem. Entre as 30 espécies coletadas nas pastagens, doze estão presentes em vegetação aberta nativa (cerrado e chaco). Isto retrata, provavelmente, um processo de colonização regional recente. Onde espécies de besouros rola-bostas, oriundas de áreas abertas, estão invadindo as pastagens amazônicas.

PALAVRAS-CHAVE: Escarabaeineos, índice de Levins, insetos copro-necrófagos, Rondônia.

\section{Dung beetles (Coleoptera: Scarabaeidae: Scarabaeinae) of forests and pastures of southwestern Brazilian Amazon: Survey of species and feeding guilds}

\begin{abstract}
The dung beetles are copro-necrophagous insects and use mainly feces and carrion of mammals as food and nesting source. These insects are sensitive to habitat modification mainly related to vegetation cover. The substitution of the Amazon forest by pasture cause drastic changes in the habitat of native species. This reduces richness and modifies assembly species composition. The aim of this work was understand the effects of substitution of native forest by pasture introduced in richness, abundance, species composition and structure of feeding guilds of dung beetle in southwestern Brazilian Amazon. A total of 10,073 individuals of dung beetles were collected, belonging to 84 species in 22 genera. Forests areas (six secondary forest fragments) had 71 species and the pastures areas (neighboring areas where original forest was substitution by introduced pasture) had significantly lower richness (30 species), and community turnover between forests and pasture was very high. In forests the majority of species were generalist, while pastures showed higher abundance of coprophagous species, which shows a change in feeding guilds caused by the substitution of forest by pasture. Among 30 species collected in pastures, twelve are present in native open vegetation too (cerrado e chaco). That represents a recent regional colonization, where species of dung beetles, coming from open areas, are invading the Amazonian pastures.
\end{abstract}

KEYWORDS: Scarabaenae beetles, index Levins, copro-necrophagous insects, Rondônia 


\section{INTRODUÇÃO}

Os besouros rola-bostas (Scarabaeidae: Scarabaeinae) são insetos detritívoros e utilizam principalmente fezes de mamíferos como fonte de alimento e nidificação. Também podem alimentar-se de carcaças e material vegetal em decomposição (Halffter e Matthews 1966). Os besouros rola-bostas desempenham importantes funçóes no ecossistema (Nichols et al. 2008), como: ciclagens de nutrientes (Bang et al. 2005); dispersão secundária de sementes (Andresen 2002) e incorporação de matéria orgânica e aeração do solo (Brussard e Slager 1986). Estes besouros também reduzem as populaçôes de dípteros hematófagos e nematoides gastrointestinais de bovinos em áreas de pastagem (Ridsdill-Smith e Edwards 2011).

Muitas espécies de besouros rola-bostas são restritas a determinados tipos de hábitat. Eles são capazes de perceber mudanças na estrutura do solo (Sowig 1995) e da vegetaçấo (Durães et al. 2005; Almeida e Louzada 2009) principalmente relacionadas a cobertura de dossel (Silva et al. 2010). Estes besouros também respondem a alteraçóes na composição da paisagem (Louzada et al. 2010). Estas mudanças na paisagem alteram a composição de espécies de besouros rola-bostas. Isto faz com que áreas vizinhas, mas com diferentes estruturas vegetacionais apresentem espécies diferentes de besouros rola-bostas (Almeida e Louzada, 2009; Silva et al. 2010). Isto ocorre principalmente entre ambientes fechados (florestas) e ambientes abertos (cerrado, chaco ou pastagem) (Spector e Ayzama 2003; Scheffler 2005).

A substituição da floresta Amazônica por pastagem está entre os principais fatores da degradação da floresta amazônica (Malhi et al. 2008). As pastagens são ambientes hostis para muitas espécies de animais (Santos-Filho et al. 2012) que não estấo adaptadas com as condiçôes adversas do ambiente aberto que apresenta baixa umidade, alta insolação e maior intensidade do vento (Laurance 2004). A substituiçấo de floresta por pastagem leva a redução na riqueza de espécies e ocasiona drástica simplificação na composiçẫo de espécies das assembleias locais (Laurance et al. 2011).

Para a região amazônica brasileira apenas quatro trabalhos relacionam os efeitos da substituição da floresta por pastagem na composiçấo de espécies de besouros rola-bostas, três para o estado do Amazonas (Klein 1989; Quintero e Roslin 2005; Quintero e Halffter 2009) e um para o estado do Pará (Scheffler 2005). Não há trabalhos para região sudoeste da Amazônia brasileira e nenhum destes trabalhos analisa a estrutura das guildas alimentares em ambiente de floresta e pastagem. Assim, o objetivo deste trabalho foi compreender os efeitos da substituição da floresta nativa por pastagem introduzida na riqueza, abundância, composição de espécies e estrutura das guildas alimentares dos besouros rola-bostas no sudoeste da Amazônia brasileira.

\section{MATERIAL E MÉTODOS}

\section{Área de estudo}

O estudo foi realizado no período chuvoso nos meses de janeiro e fevereiro de 2010. Foram delimitadas seis áreas de coleta no sudoeste da Amazônia brasileira nos município de Guajará Mirim e Nova Mamoré - RO. Cada área foi composta por um fragmento florestal e um pasto adjacente. Os fragmentos apresentam os seguintes tamanhos e coordenados: 549 ha, $10^{\circ} 37^{\prime}$ S; $64^{\circ} 59^{\prime} \mathrm{W} ; 103$ ha, $10^{\circ} 44^{\prime} S$; $65^{\circ} 17^{\prime} \mathrm{W} ; 136$ ha, $10^{\circ} 44^{\prime} \mathrm{S}$; $65^{\circ} 17^{\prime} \mathrm{W}$; 69 ha, $10^{\circ} 44^{\prime} \mathrm{S}$; $65^{\circ} 19^{\prime} \mathrm{W} ; 386$ ha, $10^{\circ} 22^{\prime} \mathrm{S}$; $64^{\circ} 44^{\prime} \mathrm{W}$ e 581 ha, $10^{\circ} 19^{\prime} \mathrm{S}$; $64^{\circ} 45^{\prime} \mathrm{W}$ respectivamente. Os fragmentos são remanescentes de floresta ombrófila aberta submontana com presença de floresta ombrófila densa submontana. O clima predominante é o equatorial quente e úmido com temperatura média anual de 24 a $26^{\circ} \mathrm{C}$. A precipitação pluviométrica anual é de 1.500 a $2.200 \mathrm{~mm}$ (IBGE 2006). As pastagens, usadas para manejo extensivo de gado de corte, sáo formadas predominantemente por plantação de Brachiaria.

\section{Amostragem dos besouros rola-bostas}

Em cada área de coleta foram delimitados 20 pontos de amostragem, dez na floresta e dez na pastagem. Os pontos, para ambos locais de coleta, foram distribuídos em dois transectos paralelos à borda com $100 \mathrm{~m}$ e $200 \mathrm{~m}$ de distância da mesma. Em cada transecto foram instalados cinco pontos de amostragem distanciados $50 \mathrm{~m}$ entre si. Em cada ponto de amostragem foram instaladas três armadilhas pitfall, equidistante três metros. Cada armadilha pitfall foi constituída por um recipiente plástico de $19 \mathrm{~cm}$ de diâmetro e $11 \mathrm{~cm}$ de profundidade. As iscas foram colocadas em recipiente plástico posicionado na borda superior da armadilha. Para a conservaçáo dos insetos foi utilizado $250 \mathrm{~mL}$ de solução salina e detergente (1\%). Para atrair os besouros rola-bostas foram utilizadas três iscas: fezes humanas, baço bovino em decomposição e fezes bovinas. As armadilhas ficaram expostas por 48 horas. No total foram instaladas 360 armadilhas, 60 por área de coleta, 30 em floresta e 30 em pasto, com dez armadilhas para cada isca por local.

Os besouros rola-bostas foram armazenados em álcool $70 \%$, triados, acondicionados em mantas entomológicas e identificados até o gênero com a chave proposta por Vaz de Mello et al. (2011). Para a identificaçáo em nível de espécie foi utilizada a coleção do Setor de Entomologia da Coleção Zoológica da Universidade Federal de Mato Grosso (CEMT), em Cuiabá - MT. Todo o material da coleta foi depositado na CEMT.

\section{Análise dos dados}

Para observar a eficiência, na captura de besouros rolabostas, das diferentes iscas foram feitas curvas de rarefaçáo 
por isca para as áreas de floresta e pastagem. Para observar a diferença na riqueza entre floresta e pastagem foram feitas curvas de rarefaçáo, com todas as iscas somadas, por habitat. Para observar o padrão de distribuição da abundância das espécies nas florestas e nas pastagens foram utilizados ranques de abundância com os dados transformados por $\log +1$. Este gráfico gera curva com o ranqueamento das espécies, da mais para a menos abundante. Para analisar a diferença na riqueza e na abundância dos besouros rola-bostas entre as florestas e pastagens foi utilizado teste t. Para validar o resultado do teste t os resíduos foram submetidos a teste de normalidade através do método de Shapiro - Wilk.

Para demonstrar o padrão da distribuição dos besouros rola-bostas nas áreas de florestas e de pastagens foi utilizado a Analise de Coordenadas Principais (PCoA). Para testar a existência de diferença significativa na composição de espécies das áreas de florestas e pastagens foi utilizado Analise de Similaridade (ANOSIM). Esta analise é considerada análoga a ANOVA, porém utiliza permutação das amostras e compara a similaridade entre matrizes de distância (Clarke 1993). Para ambas as análises foram utilizadas os dados padronizados pela abundância relativa das espécies com índice de distância de Bray-Curtis.

Para analise de amplitude do nicho alimentar das espécies foi utilizado o índice de Levins padronizado (BA). Este índice mensura a amplitude do nicho utilizado por cada espécie em escala de 0-1. Quanto mais próximo de zero mais especialista é a espécie (Krebs 1999). Foram consideradas especialistas as espécies com $\mathrm{BA}<0,5$ estas foram divididas em especialista coprófagas e necrófagas. As espécies com $\mathrm{BA} \geq 0,5$ foram consideradas generalistas. Para esta analise foram utilizadas

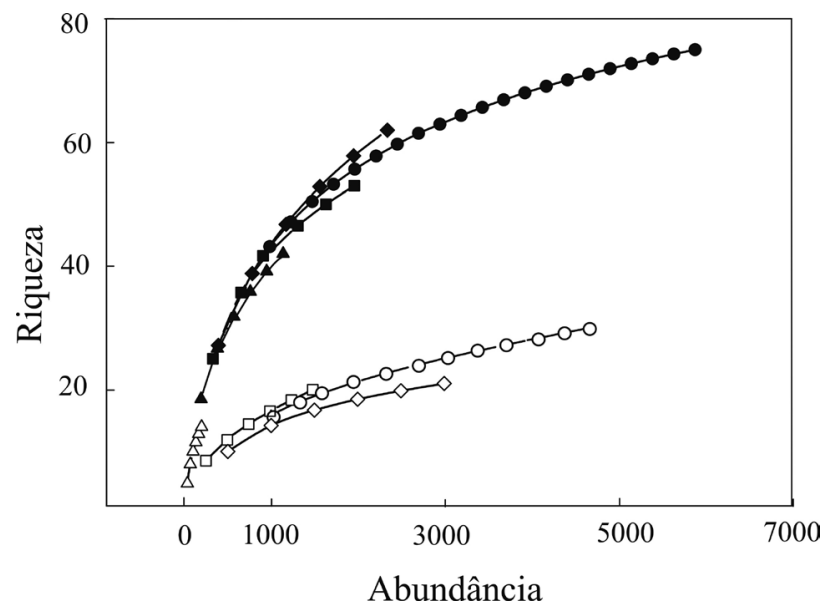

Figura 1. Curvas de rarefação para besouros rola-bostas coletados com pitfall iscados com baço bovino (quadrados), fezes humanas (losangos), fezes bovinas (triângulos) e com todas as iscas somadas (círculos) em áreas de florestas (símbolos fechados) e pastagens (símbolos abertos) no sudoeste da Amazônia brasileira, municípios de Guajará Mirim e Nova Mamoré-R0. as espécies com abundância superior a 15 indivíduos (Silva et al. 2012). As curvas de rarefação foram feitas no programa EstimateS 7.52. As demais análises foram feitas no programa R. Para fazer a matriz de distância, a PCoA, e a ANOSIM foi utilizado o pacote Vegan (Oksanen et al. 2013).

\section{RESULTADOS}

\section{Riqueza, abundância e composição de espécies}

Foram coletados 10.073 indivíduos de besouros rolabostas destes 5.870 foram coletados em florestas e 4.656 em pastagens. Quanto à riqueza foram coletadas 84 espécies distribuídas em 22 gêneros pertencentes a seis tribos: Phanaeini (11 espécies - 13\%); Canthonini (22 espécies 26\%); Oniticellini (sete espécies - 8\%); Ateuchini (11 espécies - 13\%); Coprini (24 espécies - 28\%); Onthophagini (nove espécies - 10\%). Nas florestas foram encontradas 71 espécies e nas pastagens 30 espécies. As duas áreas compartilham 16 espécies (19\%), as florestas apresentam 55 espécies exclusivas enquanto as pastagens apresentam 13 espécies exclusivas (Tabela 1).

As curvas de rarefação, tanto para floresta quanto para pastagem, não atingiram a assíntota (Figura 1). As florestas apresentaram predominância de espécies com abundância intermediária e baixa (Figura 2). As espécies mais abundantes foram Eurysternus atrosericus Génier 2009 (531 indivíduos); Canthidium sp.1 (493 indivíduos) e Dichotomius aff. lucasi (Harold 1869) (472 indivíduos) . Das 71 espécies encontradas nas florestas 32 possuíam menos de dez indivíduos, destas 11 foram singletons (Tabela 1). Para as pastagens a espécie mais abundante foi Canthon aff. simulans Martínez 1950 (2.445

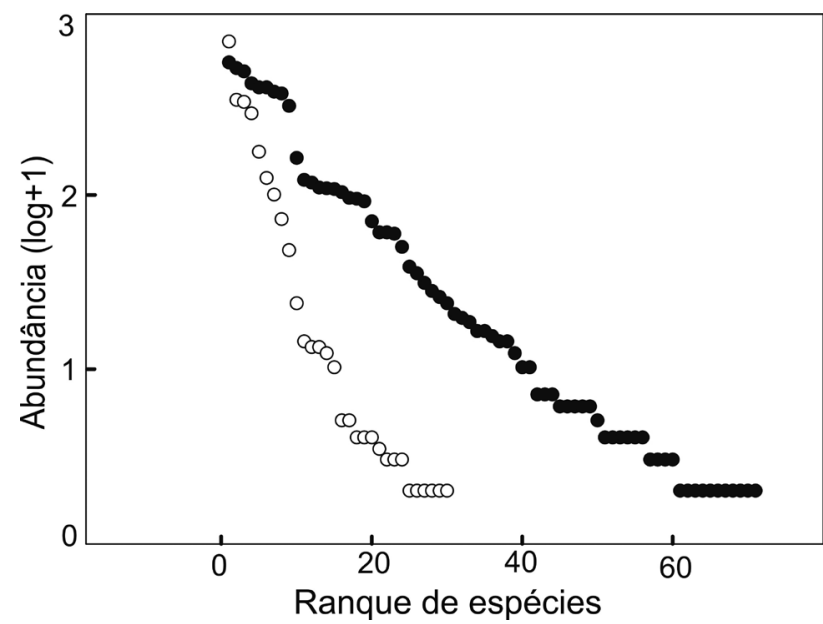

Figura 2. Ranque da abundância das espécies de besouros rola-bostas coletados com armadilhas pitfall em florestas (círculos fechados) e pastagens (círculos abertos) no sudoeste da Amazônia brasileira, municípios de Guajará Mirim e Nova Mamoré-R0, Brasil. 
Tabela 1. Guilda alimentar (GA), amplitude de nicho (BA) e abundância das espécies de besouros rola-bostas coletados com pitfall iscado com fezes humanas $(\mathrm{FH})$, baço bovino (B) e fezes bovinas (FB) em áreas de florestas e pastagens no sudoeste amazônico, municípios de Guajará Mirim e Nova Mamoré - R0 Brasil. TF= Abundância total em florestas; $\mathrm{TP}=$ Abundância total em pastagens; $\mathrm{N}=$ Necrófaga; $\mathrm{G}=\mathrm{Generalista;} \mathrm{C}=$ Coprófaga.

\begin{tabular}{|c|c|c|c|c|c|c|c|c|c|c|c|}
\hline \multirow{2}{*}{ TRIBO/ESPÉCIE } & \multicolumn{4}{|c|}{ Florestas } & \multicolumn{7}{|c|}{ Pastagens } \\
\hline & $\mathrm{B}$ & $\mathrm{FH}$ & FB & TF & $\mathrm{B}$ & $\mathrm{FH}$ & FB & TP & Total & GA & BA \\
\hline \multicolumn{12}{|l|}{ Phanaeini } \\
\hline Coprophanaeus lancifer (Linnaeus 1767) & 91 & 5 & 1 & 97 & 0 & 0 & 0 & 0 & 97 & $\mathrm{~N}$ & 0,07 \\
\hline Coprophanaeus telamon (Erichson 1847) & 21 & 11 & 1 & 33 & 0 & 0 & 0 & 0 & 33 & G & 0,47 \\
\hline Coprophanaeus jasius (Olivier 1789) & 9 & 4 & 0 & 13 & 0 & 0 & 0 & 0 & 13 & $\mathrm{~N}$ & 0,37 \\
\hline Coprophanaeus degallieri Arnaud 1997 & 0 & 1 & 0 & 1 & 0 & 0 & 0 & 0 & 1 & - & - \\
\hline Diabroctis mimas (Linné 1758) & 0 & 0 & 0 & 0 & 0 & 92 & 2 & 94 & 94 & c & 0,02 \\
\hline Oxysternon conspicillatum Weber 1801 & 49 & 52 & 1 & 102 & 0 & 0 & 0 & 0 & 102 & G & 0,52 \\
\hline Oxysternon lautum MacLeay 1819 & 3 & 0 & 0 & 3 & 0 & 0 & 0 & 0 & 3 & - & - \\
\hline Phanaeus chalcomelas (Perty 1830) & 20 & 18 & 19 & 57 & 0 & 0 & 0 & 0 & 57 & G & 1,00 \\
\hline Phanaeus bispinus Bates 1868 & 0 & 1 & 0 & 1 & 0 & 0 & 0 & 0 & 1 & - & - \\
\hline Phanaeus alvarengai Arnaud 1984 & 0 & 1 & 0 & 1 & 0 & 0 & 0 & 0 & 1 & - & - \\
\hline Phanaeus cambeforti Arnaud 1982 & 1 & 2 & 0 & 3 & 0 & 0 & 0 & 0 & 3 & - & - \\
\hline \multicolumn{12}{|l|}{ Canthonini } \\
\hline Canthon proseni Martínez 1949 & 22 & 127 & 3 & 152 & 0 & 0 & 0 & 0 & 152 & c & 0,19 \\
\hline Canthon mutabilis Lucas 1857 & 1 & 0 & 0 & 1 & 272 & 34 & 11 & 317 & 318 & $\mathrm{~N}$ & 0,17 \\
\hline Canthon aff. simulans Martínez 1950 & 7 & 1 & 1 & 9 & 914 & 1.495 & 36 & 2.445 & 2.454 & G & 0,50 \\
\hline Canthon lituratus (Germar 1813) & 0 & 0 & 0 & 0 & 17 & 632 & 49 & 698 & 698 & c & 0,11 \\
\hline Canthon aff. chalybaeus Blanchard 1846 & 0 & 0 & 0 & 0 & 19 & 2 & 1 & 22 & 22 & $\mathrm{~N}$ & 0,16 \\
\hline Canthon histrio (LePeletier e Serville 1828) & 0 & 0 & 0 & 0 & 7 & 4 & 0 & 11 & 11 & - & - \\
\hline Canthon luteicollis Erichson 1847 & 5 & 63 & 42 & 110 & 0 & 0 & 0 & 0 & 110 & G & 0,55 \\
\hline Canthon aff. angustatus Harold 1867 & 2 & 7 & 8 & 17 & 0 & 0 & 0 & 0 & 17 & G & 0,74 \\
\hline Canthon bipunctatus Burmeister 1873 & 0 & 3 & 0 & 3 & 0 & 0 & 0 & 0 & 3 & - & - \\
\hline Canthon aff. sericatus Schmidt 1922 & 1 & 0 & 0 & 1 & 0 & 0 & 0 & 0 & 1 & - & - \\
\hline Canthon semiopacus Harold 1868 & 0 & 12 & 1 & 13 & 0 & 0 & 0 & 0 & 13 & c & 0,08 \\
\hline Cryptocanthon peckorum Howden 1973 & 0 & 0 & 0 & 0 & 0 & 3 & 0 & 3 & 3 & - & - \\
\hline Deltochilum carinatum (Westwood 1837) & 9 & 2 & 0 & 11 & 0 & 0 & 0 & 0 & 11 & - & - \\
\hline Deltochilum sp. 1 & 34 & 1 & 1 & 36 & 0 & 0 & 0 & 0 & 36 & $\mathrm{~N}$ & 0,06 \\
\hline Deltochilum sp. 2 & 22 & 3 & 1 & 26 & 0 & 0 & 0 & 0 & 26 & $\mathrm{~N}$ & 0,18 \\
\hline Deltochilum sp. 3 & 244 & 42 & 15 & 301 & 0 & 0 & 0 & 0 & 301 & N & 0,24 \\
\hline Deltochilum amazonicum Bates 1887 & 16 & 3 & 0 & 19 & 0 & 0 & 0 & 0 & 19 & N & 0,18 \\
\hline Deltochilum orbiculare van Lansberge 1874 & 0 & 4 & 0 & 4 & 0 & 0 & 0 & 0 & 4 & - & - \\
\hline Pseudocanthon aff. xanthurus(Blanchard 1846) & 0 & 0 & 0 & 0 & 6 & 105 & 6 & 117 & 117 & c & 0,12 \\
\hline Canthonella sp.1 & 0 & 0 & 0 & 0 & 48 & 117 & 0 & 165 & 165 & c & 0,35 \\
\hline Sylvicanthon sp. 1 & 1 & 46 & 0 & 47 & 0 & 0 & 0 & 0 & 47 & c & 0,02 \\
\hline Hansreia sp. (Fabricius 1801) & 41 & 40 & 20 & 101 & 0 & 0 & 0 & 0 & 101 & G & 0,89 \\
\hline \multicolumn{12}{|l|}{ Oniticellini } \\
\hline Eurysternus caribaeus (Herbst 1789) & 169 & 71 & 143 & 383 & 0 & 0 & 0 & 0 & 383 & G & 0,86 \\
\hline Eurysternus wittmerorum Martinez 1988 & 43 & 9 & 34 & 86 & 1 & 0 & 0 & 1 & 87 & G & 0,73 \\
\hline Eurysternus atrosericus Génier 2009 & 169 & 224 & 138 & 531 & 0 & 0 & 0 & 0 & 531 & G & 0,94 \\
\hline Eurysternus hypocrita Balthasar 1939 & 1 & 0 & 0 & 1 & 0 & 0 & 0 & 0 & 1 & - & - \\
\hline Eurysternus arnaudi Génier 2009 & 11 & 15 & 3 & 29 & 0 & 0 & 0 & 0 & 29 & G & 0,68 \\
\hline Eurysternus strigilatus Génier 2009 & 5 & 6 & 13 & 24 & 0 & 0 & 0 & 0 & 24 & G & 0,75 \\
\hline Eurysternus hamaticollis Balthasar 1939 & 0 & 1 & 4 & 5 & 0 & 0 & 0 & 0 & 5 & - & - \\
\hline \multicolumn{12}{|l|}{ Ateuchini } \\
\hline Ateuchus aff. candezei (Harold 1868) & 263 & 106 & 35 & 404 & 0 & 0 & 0 & 0 & 404 & G & 0,50 \\
\hline Ateuchus aff. murrayi (Harold 1868) & 0 & 9 & 0 & 9 & 0 & 0 & 0 & 0 & 9 & - & - \\
\hline Ateuchus connexus(Harold 1868) & 0 & 4 & 14 & 18 & 0 & 0 & 0 & 0 & 18 & c & 0,26 \\
\hline Ateuchus aff. striatulus (Preudhomme de Borre 1886) & 1 & 11 & 78 & 90 & 0 & 0 & 1 & 1 & 91 & C & 0,17 \\
\hline
\end{tabular}


Tabela 1. Continuação.

\begin{tabular}{|c|c|c|c|c|c|c|c|c|c|c|c|}
\hline \multirow{2}{*}{ TRIBO/ESPÉCIE } & \multicolumn{4}{|c|}{ Florestas } & \multicolumn{7}{|c|}{ Pastagens } \\
\hline & $\mathrm{B}$ & $\mathrm{FH}$ & $\mathrm{FB}$ & TF & B & $\mathrm{FH}$ & FB & TP & Total & GA & BA \\
\hline Ateuchus sp. 1 & 24 & 14 & 18 & 56 & 0 & 0 & 0 & 0 & 56 & G & 0,93 \\
\hline Ateuchus sp. 2 & 33 & 209 & 112 & 354 & 0 & 0 & 0 & 0 & 354 & G & 0,59 \\
\hline Trichillum externepunctatum Preudhomme de Borre 1880 & 0 & 0 & 0 & 0 & 98 & 190 & 38 & 326 & 326 & G & 0,63 \\
\hline Eutrichillum sp. 1 & 2 & 1 & 0 & 3 & 0 & 0 & 0 & 0 & 3 & - & - \\
\hline Besourenga sp. 1 & 0 & 0 & 0 & 0 & 0 & 13 & 0 & 13 & 13 & - & - \\
\hline Uroxys sp. 1 & 1 & 2 & 0 & 3 & 0 & 2 & 0 & 2 & 5 & - & - \\
\hline Uroxys sp. 2 & 2 & 4 & 0 & 6 & 2 & 7 & 0 & 9 & 15 & G & 1,00 \\
\hline \multicolumn{12}{|l|}{ Coprini } \\
\hline Canthidium aff. cupreum (Blanchard 1846) & 23 & 246 & 114 & 383 & 1 & 2 & 0 & 3 & 386 & G & 0,51 \\
\hline Canthidium aff. gerstaeckeri Harold 1867 & 15 & 45 & 29 & 89 & 1 & 0 & 0 & 1 & 90 & G & 0,81 \\
\hline Canthidium aff. Ientum Erichson 1847 & 9 & 68 & 26 & 103 & 0 & 1 & 0 & 1 & 104 & G & 0,50 \\
\hline Canthidium aff. ardens Bates 1887 & 0 & 0 & 0 & 0 & 2 & 57 & 9 & 68 & 68 & C & 0,19 \\
\hline Canthidium aff. bokermanni (Martínez Halffter e Pereira 1964) & 1 & 1 & 0 & 2 & 0 & 0 & 0 & 0 & 2 & - & - \\
\hline Canthidium sp. 1 & 94 & 381 & 18 & 493 & 3 & 0 & 0 & 3 & 496 & C & 0,30 \\
\hline Canthidium sp. 2 & 3 & 7 & 5 & 15 & 0 & 0 & 0 & 0 & 15 & G & 0,86 \\
\hline Canthidium sp. 3 & 0 & 0 & 1 & 1 & 0 & 0 & 0 & 0 & 1 & - & - \\
\hline Canthidium sp. 4 & 0 & 0 & 1 & 1 & 1 & 0 & 3 & 4 & 5 & - & - \\
\hline Canthidium sp. 5 & 2 & 12 & 0 & 14 & 0 & 0 & 0 & 0 & 14 & - & - \\
\hline Canthidium sp. 6 & 0 & 2 & 0 & 2 & 0 & 0 & 0 & 0 & 2 & - & - \\
\hline Canthidium sp. 7 & 3 & 1 & 1 & 5 & 0 & 0 & 0 & 0 & 5 & - & - \\
\hline Canthidium sp. 8 & 0 & 0 & 5 & 5 & 0 & 0 & 0 & 0 & 5 & - & - \\
\hline Dichotomius aff. lucasi (Harold 1869) & 242 & 173 & 57 & 472 & 0 & 0 & 0 & 0 & 472 & G & 0,71 \\
\hline Dichotomius mamillatus (Felsche 1901) & 8 & 46 & 3 & 57 & 0 & 0 & 0 & 0 & 57 & C & 0,24 \\
\hline Dichotomius robustus (Luederwaldt 1935) & 4 & 2 & 0 & 6 & 0 & 0 & 0 & 0 & 6 & - & - \\
\hline Dichotomius ohausi (Luederwaldt 1923) & 0 & 3 & 0 & 3 & 0 & 0 & 0 & 0 & 3 & - & - \\
\hline Dichotomius melzeri (Luederwaldt 1922) & 0 & 5 & 1 & 6 & 0 & 0 & 0 & 0 & 6 & - & - \\
\hline Dichotomius carinatus (Luederwaldt 1925) & 2 & 3 & 0 & 5 & 0 & 0 & 0 & 0 & 5 & - & - \\
\hline Dichotomius worontzowi (Pereira 1942) & 3 & 19 & 0 & 22 & 0 & 2 & 0 & 2 & 24 & C & 0,27 \\
\hline Dichotomius aff. globulus (Felsche 1901) & 7 & 6 & 2 & 15 & 0 & 0 & 0 & 0 & 15 & G & 0,76 \\
\hline Dichotomius longiceps (Taschenberg 1870) & 0 & 0 & 0 & 0 & 1 & 0 & 0 & 1 & 1 & - & - \\
\hline Dichotomius imitator (Felsche 1901) & 0 & 2 & 0 & 2 & 0 & 0 & 0 & 0 & 2 & - & - \\
\hline Ontherus azteca Harold 1869 & 1 & 1 & 0 & 2 & 0 & 0 & 0 & 0 & 2 & - & - \\
\hline \multicolumn{12}{|l|}{ Onthophagini } \\
\hline Onthophagus aff. clypeatus Blanchard 1846 & 88 & 8 & 18 & 114 & 0 & 0 & 2 & 2 & 116 & $\mathrm{~N}$ & 0,33 \\
\hline Onthophagus aff. osculatii Guérin-Méneville 1855 & 30 & 23 & 13 & 66 & 5 & 3 & 4 & 12 & 78 & G & 1,00 \\
\hline Onthophagus aff. hirculus Mannerheim 1829 & 0 & 1 & 0 & 1 & 73 & 169 & 31 & 273 & 274 & G & 0,58 \\
\hline Onthophagus aff. onorei Zunino e Halffter 1997 & 3 & 2 & 0 & 5 & 0 & 12 & 0 & 12 & 17 & C & 0,42 \\
\hline Onthophagus aff. haemathopus Harold 1875 & 90 & 141 & 131 & 362 & 0 & 0 & 0 & 0 & 362 & G & 0,95 \\
\hline Onthophagus aff. bidentatus Drapiez 1819 & 1 & 0 & 0 & 1 & 0 & 0 & 0 & 0 & 1 & - & - \\
\hline Onthophagus aff. digitifer Boucomont 1932 & 0 & 0 & 1 & 1 & 1 & 0 & 0 & 1 & 2 & - & - \\
\hline Onthophagus aff. buculus Mannerheim 1829 & 0 & 0 & 0 & 0 & 0 & 43 & 2 & 45 & 45 & C & 0,05 \\
\hline Digitonthophagus gazella Fabricius 1787 & 0 & 0 & 0 & 0 & 0 & 0 & 4 & 4 & 4 & - & - \\
\hline Abundância & 1.952 & 2.333 & 1.132 & 5.417 & 1.472 & 2.985 & 199 & 4.656 & 10.073 & & \\
\hline Riqueza & 53 & 62 & 41 & 71 & 19 & 21 & 15 & 30 & 84 & & \\
\hline
\end{tabular}


indivíduos), seguido de Canthon lituratus (Germar 1813) (698 indivíduos). Das 30 espécies encontradas nas pastagens 15 espécies apresentaram menos de 10 indivíduos e seis foram singletons (Figura 2).

As pastagens apresentaram menor riqueza de espécies de besouros rola-bostas que as florestas $(\mathrm{t}=8,95$; $\mathrm{gl}=8,88$; $\mathrm{p}<$ $0,05)$. A abundância de besouros rola-bostas das pastagens náo diferiu da abundância encontrada nas florestas. As florestas apresentaram espécies diferentes das encontradas nas pastagens (ANOSIM: $\mathrm{R}=0,97 ; \mathrm{p}<0,05)$. Esta separação entre as florestas e as pastagens foi capturada pela PCoA que formou dois grupos distintos. O primeiro eixo da PCoA capturou $61,6 \%$ da variação na composição de espécies de besouros rolabostas. A variação na composição de espécies entre as florestas foi capturada pelo segundo eixo da PCoA que representou apenas $10 \%$ de variação na composição (Figura 3).

\section{Guildas e amplitude do nicho alimentar}

Das 84 espécies coletadas, 50 apresentaram abundância $\geq 15$. Destas 24 foram consideradas especialistas e 26 generalistas (Tabela 1). Entre as espécies especialistas 15 foram consideradas coprófagas com ocorrência ocasional em carcaças e nove foram consideradas necrófagas com ocorrência ocasional em fezes. Coprophanaeus telamon recebeu o maior valor de BA entre os especialistas $(0,47)$ dos 33 indivíduos coletados 21 foram com baço e 11 com fezes humanas e um com fezes bovinas (Tabela 1). Por isso consideramos esta espécie como generalista mesmo tendo valor de BA menor que o padronizado pela literatura.

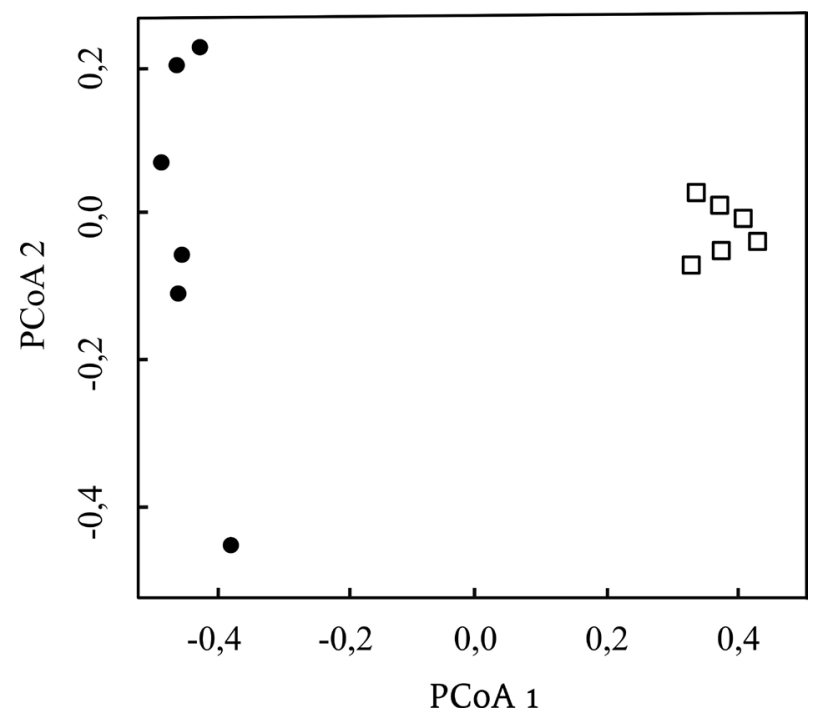

Figura 3. Ordenação dos pontos de coleta, PCoA, em função da estrutura da assembleia de besouros rola-bostas no sudoeste da Amazônia brasileira, municípios de Guajará Mirim e Nova Mamoré-R0, Brasil. Círculos fechados = áreas de florestas; quadrados abertos = áreas de pastagens. Explicação por eixo: primeiro $61,6 \%$; segundo $10 \%$.
Para as florestas 37 espécies apresentaram abundância $\geq$ 15 indivíduos, destas, 15 foram consideradas especialistas (40\%), oito coprófagas e sete necrófagas, 22 espécies foram consideradas generalistas (60\%). Para as pastagens 12 espécies apresentaram abundância $\geq 15$ indivíduos, destas nove foram especialistas (75\%), sete coprófagas e duas necrófagas e três espécies foram consideradas generalistas (25\%). Quanto à abundância as proporçôes encontradas para florestas e pastagens foram semelhantes. A maior abundância foi de generalistas (florestas 71\%; pastagens 66\%), seguida de coprófagos (florestas 16\%; pastagens 26\%) e necrófagos (florestas 11\%; pastagens 7\%).

\section{DISCUSSÃO}

\section{Riqueza, abundância e composição de espécies}

A maior riqueza de besouros rola-bostas em florestas do que em pastagens está relacionado à mudança na estrutura do habitat. A floresta amazônica apresenta maior riqueza de besouros rola-bostas quando comparada com pastagem introduzida na regiāo (Quintero e Halffter 2009; Scheffler 2005; Horgan 2007). A retirada da vegetação nativa para introdução de pastagem gera brusca mudança no habitat que ocasionam alteraçóes microclimáticas, tais como: queda na umidade e aumento da intensidade luminosa, que acarreta no ressecamento mais rápido do recurso alimentar (Klein 1989) e compactação do solo (Laurance 2004). Além da redução e modificação do tipo de recurso alimentar disponível (Laurance et al. 2011). Estes fatores exercem efeito na distribuição espacial dos besouros rola-bostas e influenciam na escolha e na preferência do habitat pelas espécies (Silva et al. 2010).

Assim, poucas espécies de besouros rola-bostas amazônicos conseguem utilizar de forma eficiente as pastagens como habitat e as fezes bovinas como alimento e local para nidificação. Isto acarreta modificação do padrão de distribuição da abundância caracterizada principalmente pelo surgimento de espécies com elevada abundância (Quintero e Halffter 2009) e redução no número de espécies (Estrada et al. 1998; Scheffler 2005).

A baixa similaridade na composição de espécies de besouros rola-bostas entre as áreas de florestas e as pastagens amazônicas é esperada. As pastagens apresentaram 13 espécies exclusivas $(43,3 \%)$ muitas destas espécies são registradas para o cerrado: Onthophagus aff. hirculus, O. buculus, Canthon mutabilis, C. lituratus, C. aff. chalybaeus, Pseudocanthon aff. xanthurum, Diabroctis mimas e Trichillum externepunctatum são espécies com distribuição para áreas abertas e secas como cerrado e caatinga (Almeida e Louzada 2009; Costa et al. 2009; Silva et al. 2012). Digitonthophagus gazella foi introduzida no Brasil na década de 80 para controle da mosca do chifre, mas é originária das savanas africanas, esta espécie é comumente registrada para áreas de pastagem (Silva e Vidal 2007) e savanas 
Intra-Amazônicas (Matavelli e Louzada 2008). Com isso podemos inferir que as pastagens do sudoeste da Amazônia brasileira foram colonizadas por espécies oriundas de áreas de Cerrado. Os ambientes secos são os principais fornecedores de espécies para as pastagens na América do Sul com forte processo de colonização regional recente.

\section{Guildas e amplitude do nicho alimentar}

O elevado número de espécies generalistas para as florestas é esperado para besouros rola-bostas na região neotropical. Os besouros rola-bostas neotropicais apresentam grande variação de hábitos alimentares podendo alimentarse de fezes, principalmente de mamíferos, carcaça e fruto em decomposição, fungos e apresentar comportamento de predação (Halffter e Matthews 1966). Esta radiação adaptativa ocorreu durante o Pleistoceno impulsionado pela extinção em massa dos grandes mamíferos que ocasionou em drástica redução na disponibilidade de fezes. Este aumento na competição pelo recurso permitiu que novos mecanismos de obtenção de alimento fossem selecionados e beneficiados proporcionando a diversificação dos hábitos alimentares dos besouros rola-bostas neotropicais (Halffter e Edmonds 1982).

Entre as espécies especialistas necrófagas coletados em florestas neste trabalho, Coprophanaeus lancifer (Linnaeus 1767) é registrada como uma das maiores espécies de besouros necrófagos amazônicos (Mise et al. 2010; UrurahyRodrigues et al. 2008). C. jasius (Olivier 1789) também é considerada um grande besouro necrófago amazônico, principalmente para cadáveres recentes (Halffter e Matthews 1966). O gênero Deltochilum é fundamentalmente necrófago encontrado regularmente em carcaça de animais, porém pode ser capturado com armadilhas iscadas com fezes e com fungo (Halffter e Matthews 1966). As espécies necrófagas encontradas neste trabalho em áreas de pastagens, Canthon aff. chalybaeus e C. mutabilis, também foram encontrada em maior abundância em armadilhas iscadas com carcaças do que com fezes humanas nos campos sulinos (Silva et al. 2012).

A maior proporção de coprófagos nas pastagens é esperada devido maior disponibilidade de fezes bovina como recurso alimentar e para nidificação. As pastagens são habitats simples e homogêneos com baixa diversidade de recurso alimentar. Esta simplificação do habitat e homogeneização dos recursos alimentares acarretou na elevação da abundância de poucas espécies que demostraram melhores condiçóes adaptativas as condiçóes microclimáticas das pastagens e conseguiram utilizar as fezes bovinas como fonte de alimento e nidificação.

\section{CONCLUSÃO}

A retirada da floresta e introdução de pastagem ocasionou redução na riqueza e modificação na composição de espécies. A composiçáo de espécies das pastagens apresentou grande influência de espécies oriundas de áreas abertas (cerrado), demostrando um processo de colonização regional recente. A drástica redução na riqueza e a colonização de espécies oriundas do cerrado demonstra a baixa capacidade das espécies nativas de utilizarem os pastos introduzidos na regiáo amazônica. A floresta, além de suportar maior riqueza de besouros rolabostas, abriga maior quantidade de espécies generalistas. As pastagens apresentaram mais espécies coprófagas o que demonstra uma modificação na guilda alimentar causado pela substituição da floresta por pastagem.

\section{AGRADECIMENTOS}

Nós agradecemos a Fundação de Amparo a Pesquisa do Estado de Mato Grosso (FAPEMAT- 756935/2011) e o Conselho Nacional de Desenvolvimento Científico e tecnológico (CNPq - 304925/2010-1, 302997/2013-0, 405697/2013-9, 484035/2013-4, 202327/2013-2) pelo aporte financeiro. Agradecemos a Ana Silvia Tissiani, Gimo M. Daniel e aos revisores anônimos pelas contribuiçôes no manuscrito.

\section{BIBLIOGRAFIA CITADA}

Almeida, S.S.P.; Louzada, J.N.C. 2009. Estrutura da comunidade de Scarabaeinae (Scarabaeidae: Coleóptera) em fitofisionomias do cerrado e sua importância para a conservação. Neotropical Entomology, 38: 32-43.

Andresen, E. 2002. Dung beetles in a Central Amazonian rainforest and their ecological role as secondary seed dispersers. Ecological Entomology, 27: 257-270.

Bang, H.S.; Lee, J.H.; Kwon, O.S.; Na, Y.E.; Jang, Y.S.; Kim, W.H. 2005. Effects of paracoprid dung beetles (Coleoptera: Scarabaeidae) on the growth of pasture herbage and on the underlying soil. Applied Soil Ecology, 29: 165-171.

Brussaard, L.; Slager, S. 1986. The influence of soil bulk density and soil moisture on the habitat selection of the dung beetle Typhaeus typhoeus in the Netherlands. Biology and Fertility of Soils, 2: 51-58.

Clarke, K. R. 1993. Non-parametric multivariate analyses of changes in community structure. Australian Journal of Ecology, 18: 117-143.

Costa, C.M.Q.; Silva, F.A.B.; Farias, Â.I.; Moura, R.C. 2009. Diversidade de Scarabaeinae (Coleoptera, Scarabaeidae) coletados com armadilha de interceptação de vôo no Refúgio Ecológico Charles Darwin, Igarassu-PE, Brasil. Revista Brasileira de Entomologia, 53: 88-94.

Durães, R.; Martins, W.P.; Vaz-de-Mello, F.Z. 2005. Ecology, behavior and bionomics dung beetle (Coleoptera: Scarabaeidae) assemblages across a natural forest-cerrado ecotone in Minas Gerais, Brazil. Neotropical Entomology, 34: 1-11.

Estrada, A.; Coates-Estrada, R.; Dadda, A.C.; Cammarano, P. 1998. Dung and carrion beetles in tropical rain forest fragments and agricultural habitats at Los Tuxtlas, Mexico. Journal of Tropical Ecology, 14: 577-593. 
Halffter, G.; Edmonds, W.D. 1982. The nesting behavior of dung beetles (Scarabaeinae): an ecological and evolutive approach. Unesco, instituto ecologia, México, 155p.

Halffter, G.; Matthews, E.G. 1966. The natural history of dung beetles of the subfamily Scarabaeinae (Coleoptera, Scarabaeidae). Folia Entomologica Mexicana, 12: 1-312.

Horgan, F. 2007. Dung beetles in pasture landscapes of Central America: proliferation of synanthropogenic species and decline of forest specialists. Biodiversity and Conservation, 16: 2149-2165.

IBGE, 2006. Mapa de vegetação do estado de Rondônia. (geoftp. ibge.gov.br/mapas/tematicos/tematico_estadual/RO_vegetacao. pdf). Acesso em 01/10/2009.

Klein, B.C. 1989. Effects of forest fragmentation on dung and carrion beetle communities in Central Amazônia. Ecology, 70: 1715-1725.

Krebs, C.J. 1999. Ecological Methodology. California, Benjamin Cummings, 620p.

Laurance, W.F. 2004. Forest-climate interactions in fragmented tropical Landscapes. Philosophical Transactions of The Royal Society, 159: 345-352.

Laurance, W.F.; Camargo, J.L.C.; Luizão, R.C.C.; Laurance, S.G.; Pimm, S.L.; Bruna, E.M.; et al. 2011. The fate of Amazonian forest fragments: a 32-year investigation. Biological Conservation, 144: 56-67.

Louzada, J.; Lima, A.P.; Matavelli, R.; Zambaldi, L.; Barlow, J. 2010. Community structure of dung beetles in Amazonian savannas: role of fire disturbance, vegetation and landscape structure. Landscape ecology, 25: 631-641.

Malhi, Y.; Roberts, T.; Betts, R.A.; Killeen, T.J.; Li, W.; Nobre, C. 2008. Climate Change, Deforestation, and the Fate of the Amazon. Science, 319: 169-172.

Matavelli, R.A.; Louzada, J.N.C. 2008. Invasão de áreas de savana intra-amazônicas por Digitonthophagus gazella (Fabricius, 1787) (Insecta: Coleoptera: Scarabaeidae). Acta Amazonica, 38: 153-158.

Mise, K.M.; Souza, A.S.B.; Campos, C.M.; Keppler, R.L.F.; Almeida, L.M. 2010. Coleoptera associados a carcaça de porco em reserva florestal, Manaus, Amazonas, Brazil. Biota Neotropical, 10: 321-324.

Nichols, E.; Spector, S.; Louzada, J.; Larsen, T.; Amezquita, S.; Favila, M.E. 2008. Ecological functions and ecosystem services provided by Scarabaeinae dung beetles. Biological Conservation, 141: 1461-1474.

Oksanen, J.; Blanchet, F.G.; Kindt, R.; Legendre, P.; Minchin, P.R.; O'Hara, R.B. ; et al. 2013. Vegan: Community Ecology Package. R package version 2.0-8. http://CRAN.R-project.org/ package= =egan.
Quintero, I.; Halffter, G. 2009. Temporal changes in a community of dung beetles (Insecta: Coleoptera: Scarabaeinae) resulting from the modification and fragmentation of tropical rain forest. Acta Zoológica Mexicana, 25: 625-649.

Quintero, I.; Roslin, T. 2005. Rapid recovery of dung beetle communities following habitat fragmentation in Central Amazonia. Ecology, 86: 3303-3311.

Ridsdill-Smith, T.J.; Edwards, P.B. 2011. Biological control: ecosystem functions provided by dung beetles. In. Simmons, W.; Ridsdill-Smith, T.J. (Ed.) Ecology and evolution of dung beetles. Wiley-Blackwell Publishing Ltd., Oxford, p. 245-264.

Santos-Filho, M.; Peres, C.A.; Silva, D.J.; Sanaiotti, T.M. 2012. Habitat patch and matrix effects on small-mammal persistence in Amazonian forest fragments. Biodiversity and Conservation, 21:1127-1147.

Scheffler, P.Y. 2005. Dung beetle (Coleoptera: Scarabaeidae) diversity and community structure across three disturbance regimes in eastern Amazonia. Journal of Tropical Ecology, 21: 9-19.

Silva, P.G.; Vidal, M.B. 2007. Atuação dos escarabeídeos fimícolas (Coleoptera: Scarabaeidae sensu stricto) em áreas de pecuária: potencial benéfico para o município de Bagé, Rio Grande do Sul, Brasil. Revista de Ciências Agroveterinárias, 6: 162-169.

Silva, P.G.; Audino, L.D.; Nogueira, J.M.; Moraes, L.P.; Vaz-deMello, F.Z. 2012. Escarabeineos (Coleoptera: Scarabaeidae: Scarabaeinae) de uma área de campo nativo no bioma Pampa, Rio Grande do Sul, Brasil. Biota Neotropical, 12: 1-9.

Silva, R.J.; Diniz. S.; Vaz-de-Mello, F.Z. 2010. Heterogeneidade do habitat, riqueza e estrutura da assembléia de besouros rola-bostas (Scarabaeidae: Scarabaeinae) em áreas de cerrado na Chapada dos Parecis, MT. Neotropical Entomology, 39: 934-940.

Sowig, P. 1995. Habitat selection and offspring survival rate in three paracoprid dung beetles: the influence of soil type and soil moisture. Ecography, 18: 147-154.

Spector, S.; Ayzama, S. 2003. Rapid turnover and edge effects in dung beetle assemblages (Scarabaeidae) at a Bolivian neotropical forest - savanna ecotone. Biotropica, 35: 394-404.

Ururahy-Rodrigues, A.; Rafael, J.A.; Wanderley, R.F.; Marques, H.; Pujol-Luz, J.R.; Henriques, A.L. et al. 2008. Coprophanaeus lancifer (Linnaeus, 1767) (Coleoptera, Scarabaeidae) activity moves a man-size pig carcass: Relevant data for forensic taphonomy. Forensic Science International, 182: 19-22.

Vaz-de-Mello, F.Z.; Edmonds, W.D.; Ocampo, F.; Schoolmeesters, Paul. 2011. A multilingual key to the genera and subgenera of the subfamily Scarabaeinae of the New World. Zootaxa, 2854: 1-73.

Recebido em 05/09/2013

Aceito em 12/02/2014 